\title{
IMPLEMENTAÇÃO DO ALGORITMO DE CONTROLE PID EM PLATAFORMA VBA PARA APLICAÇÃO EM PLANTAS DE PROCESSO DO SIMULADOR ASPEN DYNAMICS
}

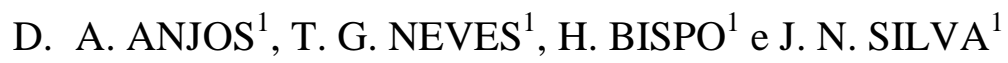 \\ ${ }^{1}$ Universidade Federal de Campina Grande, Unidade Acadêmica de Engenharia Química \\ E-mail para contato: nilton@ deq.ufcg.edu.br
}

\begin{abstract}
RESUMO - O controle automático tem sido fundamental para o desenvolvimento da automação. O intuito da utilização de controle em processos consiste em atuar sobre as condições a que o processo está sujeito, na tentativa de atingir o mais próximo possível do ponto desejado. Esse trabalho tem por objetivo implementar o algoritmo clássico de controle PID e as estratégias clássicas de sintonia em plataforma $V B A$, no intuito de aplicação de sistemas de controle em plantas de processos, utilizando um sistema integrado com Aspen Dynamic ${ }^{\circledR}$. Para avaliação da ferramenta, foi utilizada uma planta de processo composta por colunas de destilação de uma unidade de tratamento de Nafta modelada em Aspen Dynamic ${ }^{\circledR}$, à mesma previamente validada com dados reais de planta. A ferramenta desenvolvida na plataforma VBA foi comparada com a disponível na plataforma Aspen Dynamic ${ }^{\circledR}$ utilizado na pesquisa, a qual mostrou desempenho final satisfatório.
\end{abstract}

\section{INTRODUÇÃO}

Sistemas dinâmicos tais como plantas industriais estão sujeitos a perturbações diversas, e por isso se faz necessário controladores para que possam operar dentro das especificações desejadas. De forma geral, é importante que o processo esteja sempre próximo de um dado ponto estacionário de operação, sendo o papel dos controladores minimizar os efeitos externos que tentam desviá-lo desta condição. Os pontos de operação podem ser definidos a requisitos de qualidade, segurança, redução do impacto ambiental, adaptação às restrições inerentes ao processo, otimização ou resultado econômico do processo.

De acordo com Luyben e Luyben (1997) existem quatro níveis de controle de processo. Movendo-se estes níveis aumenta a importância, o impacto econômico e as oportunidades para engenheiros de controle de processo para fazer contribuições significativas. O nível mais baixo é o ajuste do controlador. O segundo nível é algorítmico, decidir que tipo de controlador usar (P, PI, PID, multivariado, modelo preditivo, etc.). $\mathrm{O}$ terceiro nível é a estrutura de controle do sistema, que terá o papel de determinar o que controlar e o que manipular, esse nível é conhecido como "emparelhamento". O nível superior é um processo de projeto. Do fluxograma do processo utilizam-se os parâmetros de projeto que produzem uma planta facilmente controlável.

Este trabalho tem por objetivo a implementação, em plataforma $V B A$, do algoritmo clássico de sistema de controlador PID, para utilização em plantas modeladas no simulador 
Aspen Dynamics® no intuito de criar um ambiente de treinamento de sistemas de controle e operacionalidade de plantas de processo.

\section{REVISÃO BIBLIOGRÁFICA}

\subsection{Modelagem e Controle de Processos}

Controlar um processo significa atuar sobre ele de modo a atingir um objetivo desejado (Ogata, 1982). Geralmente o objetivo é manter o processo sempre próximo de um dado valor estacionário (set point), através da manipulação de certas variáveis para ajustar as variáveis de interesse, com o intuito de minimizar os efeitos externos que tentam desviá-lo desta condição. Este estado estacionário pode ter sido escolhido por atender melhor aos requisitos de qualidade, segurança do processo, satisfazer as restrições ambientais, adaptação às restrições inerentes ao processo, otimização, uso mais eficientes de matérias-primas e energia ou aumento da rentabilidade (Luyben, Luyben, 1997; Stephanopoulos, 1984; Coughanowr e Leblanc, 2009).

Para desenvolvimento de projetos sistemas de controle, a modelagem dinâmica é fundamental. Trata-se de uma representação matemática do processo que pode ser determinada a partir da aplicação de equações de balanços de massa, energia e quantidade de movimento e de equações constitutivas (Stephanopoulos, 1984). O comportamento da dinâmica do processo pode ser obtido através das funções de transferências do sistema. Segundo Coughanowr e LeBlanc (2009), uma função de transferência relaciona duas variáveis em um processo físico, umas destas é a causa (função de força ou variável de entrada), e a outra é o efeito (resposta ou variável de saída). A Equação 1 representa matematicamente a forma genérica de uma função de transferência, que é apresentada em forma de diagrama de blocos na Figura 1.

$$
G(s)=\frac{Y(s)}{X(s)}=\frac{a_{n} s^{n}+a_{n-1} s^{n-1}+\ldots+a_{0} s^{0}}{b_{m} s^{m}+b_{m-1} s^{m-1}+\ldots+b_{0} s^{0}} e^{-s \theta}, m \geq n
$$

onde: $G(s)$ é a função de transferência, expressando como a saída $X(s)$ se comporta com a entrada $Y(s)$.

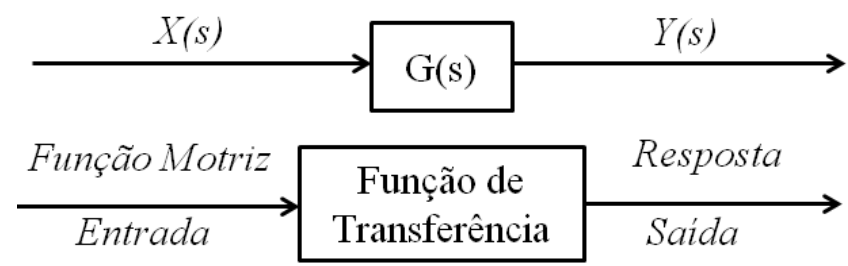

Figura 1 - Diagrama de blocos para uma função de transferência genérica.

\subsection{Leis de controle e a Estratégia Feedback}

Uma lei de controle é um conjunto de regras que são utilizadas para determinar os comandos a serem enviados a um sistema baseado no estado desejado de um sistema. Leis de controle são utilizadas, por exemplo, para ditar como uma temperatura de um reator é 
corrigida para uma temperatura desejada, definida por set point, enviando comandos para um atuador. Existem várias leis fundamentais que têm sido desenvolvidas na área de controle de processos, sendo estas, resultado de muitos anos de experiência. Segundo Luyben (1990), as leis básicas de controle são:

i) Primeira Lei: “O sistema de controle mais simples que irá fazer o trabalho é o melhor”.

ii) Segunda Lei: "Deve-se entender o processo antes que se possa controlá-lo".

A literatura apresenta diversas estratégias de controle de processos, que é aplicada de acordo com a necessidade de melhores objetivos de controle ou de exigências do processo. Dentre as estratégias conhecidas, a aplicação de controladores por retroalimentação (feedback) é a mais comum, que consiste da comparação do resultado obtido na saída do processo com o valor desejado (set point) (Svrcek, et. al. 2006). A Figura 2 apresenta a estrutura feedback em diagrama de blocos.

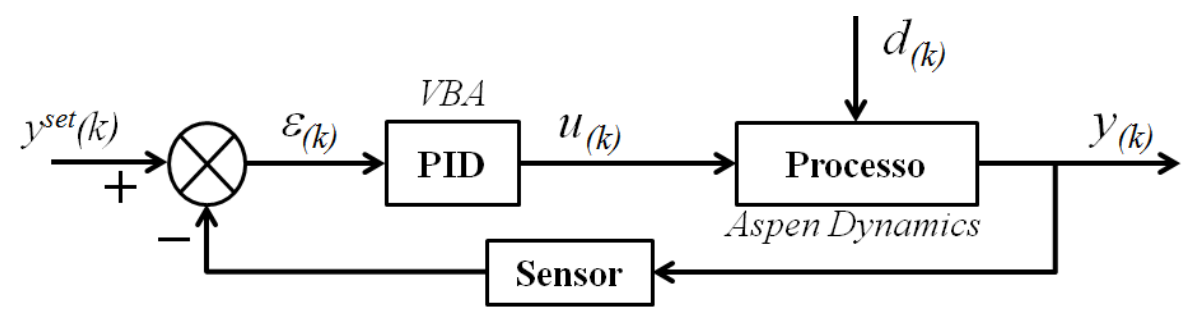

Figura 2 - Sistema de Controle de Processo feedback.

Segundo Stephanopoulos (1984), a estratégia feedback, consiste de três etapas: (1) medidas da variável controlada, $y_{m}$, são coletadas usando um equipamento de medida apropriado; (2) o valor da variável controlada é comparado com o valor de set point, $y_{S P}$, e o desvio (erro, $\varepsilon$ ) é calculado como sendo $\varepsilon=y_{S P}-y_{m}$; (3) o valor do desvio $\varepsilon$ é fornecido para o controlador principal. O controlador, por sua vez altera o valor da variável manipulada, $m$, de modo a reduzir a magnitude do desvio $\varepsilon$. Normalmente, o controlador não afeta a variável manipulada diretamente, mas através de outro dispositivo, conhecido como elemento de controle final (normalmente uma válvula).

\subsection{Controladores PID}

Em 1935, Ralph Clarridge da Taylor Instrument Companies criou o controlador de três termos, ao utilizar um controlador que antecipava a variação no sinal de erro para solucionar um problema de oscilação de uma malha de controle de temperatura em uma indústria de celulose. Chamada inicialmente de pré-act, a ação derivativa foi testada apenas em casos especiais até o ano de 1939, quando uma versão totalmente reprojetada do controlador Proporcional Integral Derivativo, o PID Fulscope foi oferecida como padrão nos sistemas de controle comerciais da empresa (Aström, 2012).

O controlador PID é normalmente aplicado para sistemas SISO (simple-input and simple output), em que a ação do controlador atua diretamente em uma variável manipulada (MV) para ajustar uma variável de processo (PV), onde as mesmas estão relacionadas através 
de um modelo representativo do processo (Svrcek et al., 2006). A equação clássica do PID no domínio tempo pode ser apresentada por:

$$
f_{c}(t)=k c e(t)+\frac{k c}{\tau i} \int_{0}^{\infty} e(t) d t+k c \tau d \frac{d e(t)}{d t}+f_{s}
$$

onde: $f_{c}(t)$ é saída do controlador e $f_{s}$ o valor estacionário de referência, $k_{c}, \tau_{i}$ e $\tau_{d}$ são as constantes proporcional, tempo integral e tempo derivativo, respectivamente e $e(t)$ é o desvio entre o processo e setpoint.

A implementação digital da Equação 2, contínua no domínio do tempo, requer a sua discretização. Tomando um intervalo de tempo $\Delta t$, que traduz a periodicidade com que o controlador calcula uma nova ordem de comando, sendo o índice $k$ que representa o instante de tempo e corresponde ao instante em que o valor $t$, de um modo genérico, o erro nos dois instantes anteriores, $e_{k-1}$ e $e_{k-2}$ pode ser expresso por:

$$
\int_{t_{k-2}}^{t_{k-1}} e(t) d t \cong\left[e\left(t_{k-2}\right) \cdot\left(t_{k-1}-t_{k-2}\right)+\frac{\left(e\left(t_{k-1}\right)+e\left(t_{k-2}\right)\right)}{2}\right]
$$

Da mesma forma o erro nos dois instantes anteriores, $e_{k}$ e $e_{k-1}$, podem ser expressos por:

$$
\int_{t_{k-1}}^{t_{k}} e_{(t)} d t \cong\left[e\left(t_{k-1}\right) \cdot\left(t_{k}-t_{k-1}\right)+\frac{\left(e\left(t_{k}\right)+e\left(t_{k-1}\right)\right.}{2}\right]
$$

As parcelas das equações 3 e 4 estão mostradas na figura 3 .

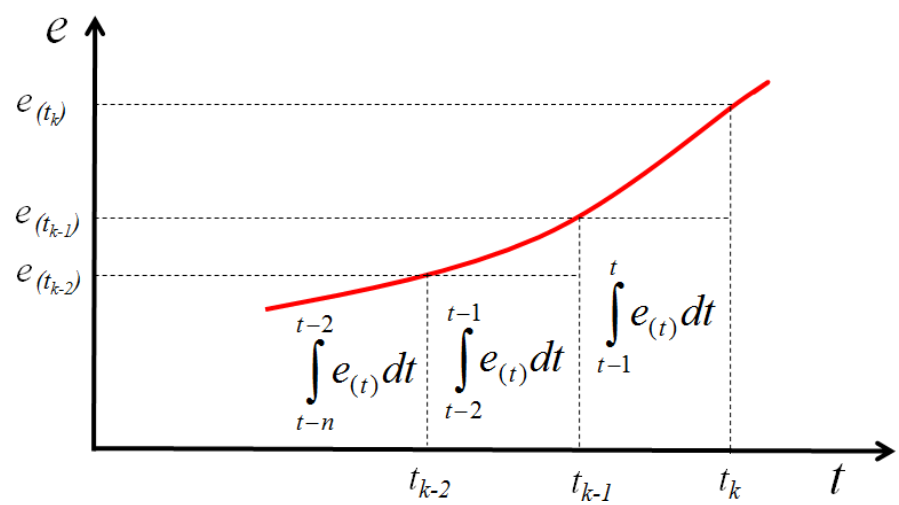

Figura 3 - Ilustração dos intervalos de discretização.

Pode-se escrever a Equação 2 na forma discreta para o ponto $k$ a $k-1$ como segue:

$$
m_{c\left(t_{k}\right)}=k_{c} e_{\left(t_{k}\right)}+\frac{k_{c}}{\tau_{i}} \sum_{k-n}^{k} e_{(t)} \Delta t+k_{c} \tau_{d} \frac{\left(e_{\left(t_{k}\right)}-e_{\left(t_{k}-1\right)}\right)}{\Delta t}
$$




$$
m_{c\left(t_{k}-1\right)}=k_{c} e\left(t_{k-1}\right)+\frac{k_{c}}{\tau_{i}} \sum_{k-n}^{k-1} e_{(t)} \Delta t+k_{c} \tau_{d} \frac{\left(e_{\left(t_{k}-1\right)}-e_{\left(t_{k}-2\right)}\right)}{\Delta t}
$$

A variação entre os pontos $k$ a $k-1$ pode ser escrita por:

$$
\Delta m_{c}=k_{c}\left(e\left(t_{k}\right)-e\left(t_{k-1}\right)\right)+\frac{k_{c}}{\tau_{i}}\left[\frac{\left(e\left(t_{k}\right)+e\left(t_{k-1}\right)\right.}{2}\right] \Delta t+k_{c} \tau_{d} \frac{\left[e\left(t_{k}\right)-2 e\left(t_{k-1}\right)+e\left(t_{k-2}\right)\right]}{\Delta t}
$$

A equação 7 é uma das equações digitais que possibilita implementar a lei de controle PID em um computador ou microprocessador.

\section{METODOLOGIA}

\subsection{Coluna depentanizadora de nafta}

Uma unidade de tratamento de nafta foi utilizada no presente trabalho, sendo a mesma modelada na plataforma Aspen Dynamics ${ }^{\circledR}$, previamente validada com dados reais de planta de uma indústria. Para avaliar a estrutura da auto-sintonia proposta neste trabalho, uma coluna de destilação depentanizadora foi considerada, como mostrado no diagrama simplificado na Figura 4.

A coluna adotada para o estudo é constituída por 37 estágios, com uma carga de alimentação média de $57,55 \mathrm{t} \mathrm{h}^{-1}$, composta de $\mathrm{C} 1$ a $\mathrm{C} 11$ de compostos parafínicos e aromáticos mais representativos da carga real do processo. A finalidade desta coluna é separar os componentes maiores que C5 na corrente de base e os menores e iguais a C5 na corrente de destilado.

A corrente de produto de topo, identificada como 454, é composta de 0,998 de hidrocarbonetos até o C5. A corrente do produto de base, identificada como 471, sendo composta de 0,999 de hidrocarbonetos maiores que o C5.

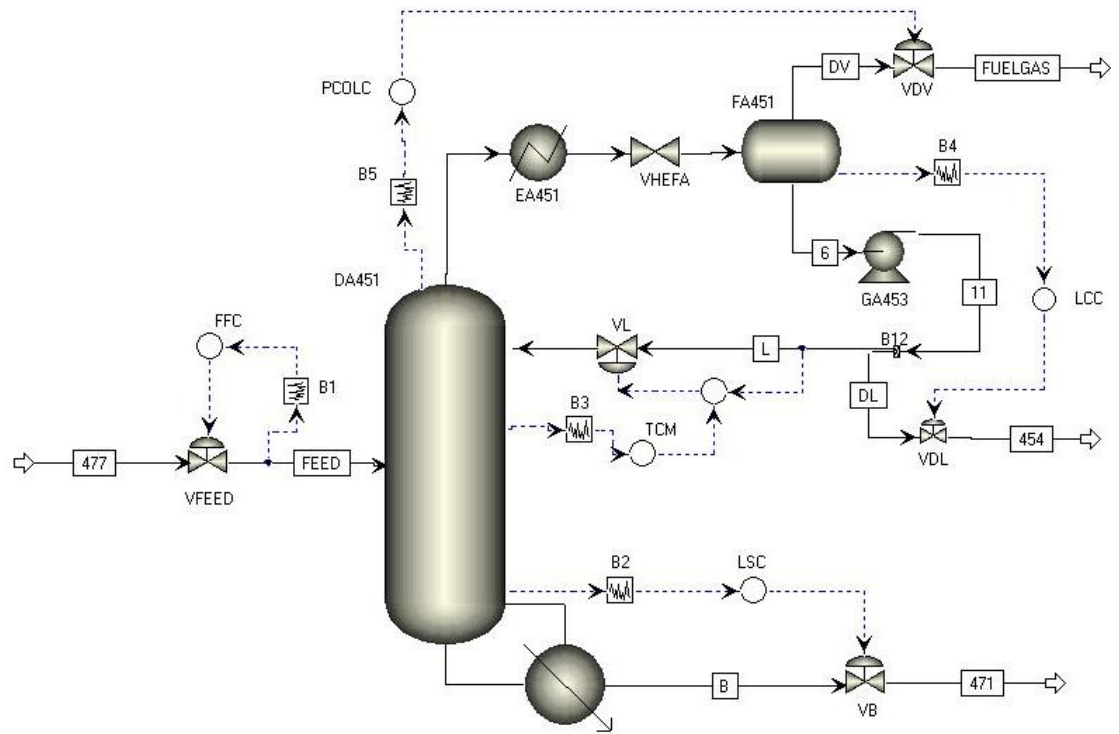

Figura 4 - Fluxograma da coluna de destilação e malhas de controle no Aspen Dynamics ${ }^{\circledR}$. 


\subsection{Algoritmo PID para a plataforma VBA}

O algoritmo de controle PID foi implementado em plataforma VBA, e sua forma discretizada representada pela Equação 7, de acordo com o diagrama de blocos apresentado na Figura 4. A partir da planta implementada na plataforma Aspen Dynamics ${ }^{\circledR}$, a conexão com VBA foi realizada utilizando a tecnologia de comunicação Object Linking and Embedding (OLE). Dessa forma, a ferramenta foi estruturada considerando o algoritmo simplificado representado na Figura 4.

Inicialmente com a comunicação entre o Aspen® Dynamics estabelecida, informações são fornecidas através da planilha e o controlador PID conectado ao processo. Com o intuito de avaliar o desempenho de atuação do controlador sobre o processo, aplica-se um distúrbio no set point, avaliando assim a atuação do controlador sobre o sistema.

O acesso as variáveis internas na simulação foi possível considerando a linha de código padrão como mostra a Tabela 1. A string path é referente ao caminho do arquivo, enquanto Object Variable correspondente a variável ao acesso de entrada ou saída no Aspen®.

Tabela 1 - Código de comunicação entre VBA e Aspen $®$ Dynamics

\begin{tabular}{l|l}
\hline Acesso In/Output & $=$ 'Aspen Dynamics Language'|'path *.dynf'!'\Object Variable.Value' \\
\hline
\end{tabular}

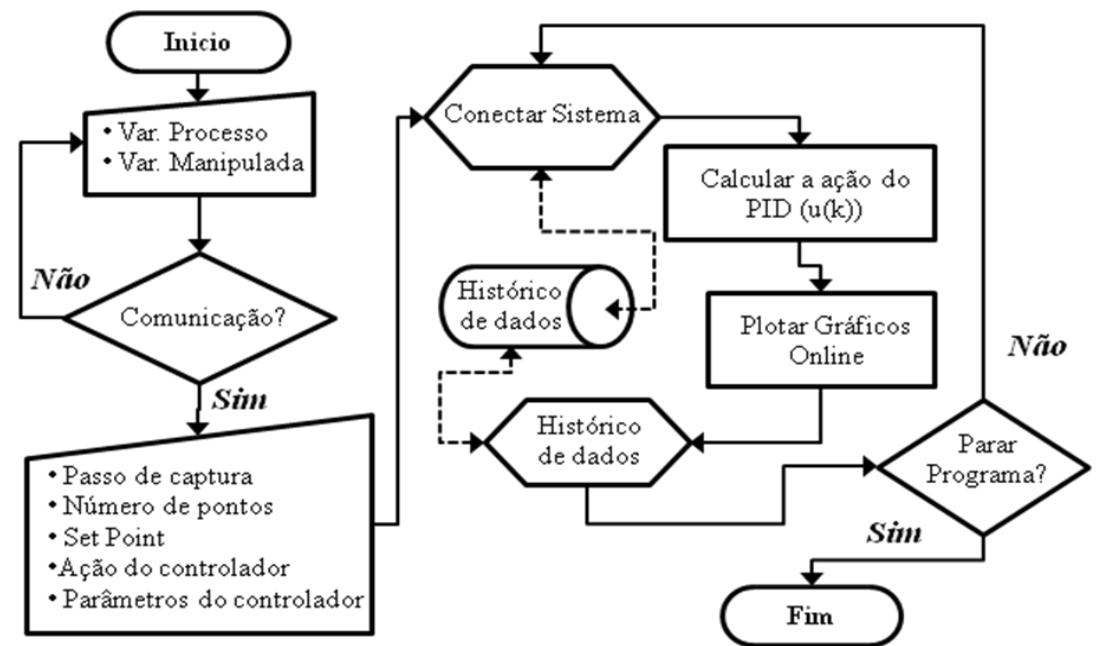

Figura 4 - Algoritmo simplificado do programa do controlador PID desenvolvido no VBA

\section{RESULTADOS E DISCUSSÃO}

\subsection{Interface da ferramenta PID}

A ferramenta foi desenvolvida em uma interface diretamente estruturada em planilhas Excel ${ }^{\circledR}$ como mostra a Figura 5. A interface apresenta as etapas do algoritmo apresentado na figura 4, onde os códigos, em VBA, correspondentes às mesmas são executados a partir de macros com as chamadas dos eventos pelos respectivos botões. 


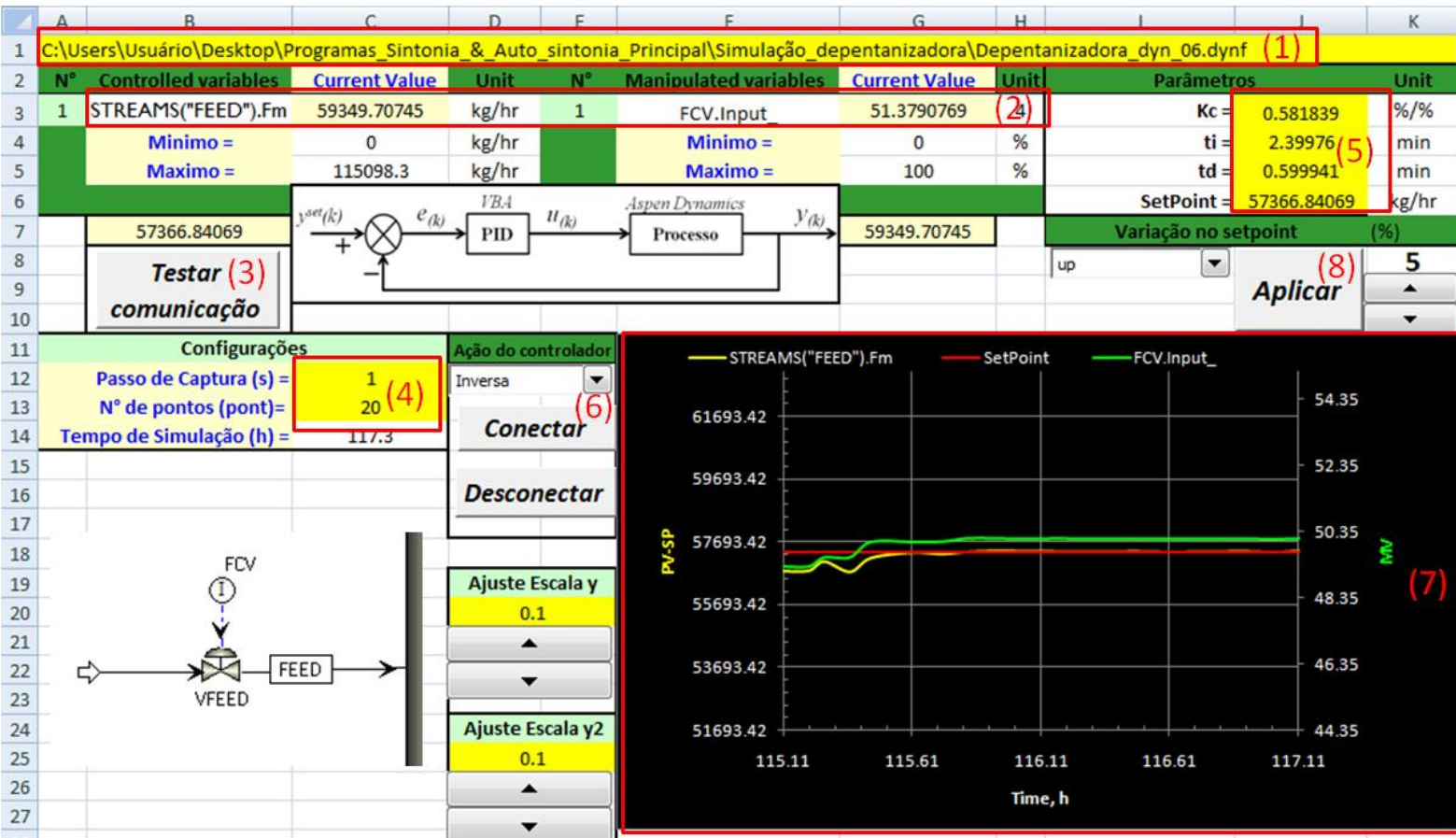

Figura 5 - Interface da planilha do Algoritmo do PID.

É possível observar que a interface de aplicação da ferramenta é bastante simples, tornando-se uma ferramenta que pode ser facilmente aplicada em diversas análises.

A etapa inicial, (1), consiste em definir o local do arquivo da simulação dinâmica do Aspen ${ }^{\circledR}$ Dynamics. Seguido pela definição das variáveis de acesso, (2), ou seja, das variáveis de processo e manipuladas, como exemplificado para a malha de vazão de alimentação na mesma figura.

A etapa (3) consiste na verificação da conexão das variáveis. Caso as variáveis estiverem escritas de forma incorreta se faz necessário corrigir as mesmas. Ainda referente à conexão, a etapa (4) permite especificar o passo de captura e o número de pontos a ser mostrado no gráfico de visualização das variáveis. E na etapa (5) corresponte a especificação dos parâmetros de sintonia, que podem ser obtidos pelos métodos clássicos de sintonia.

Na etapa (6) corresponde a conexão do sistema de forma on-line. Seguido dessa forma pela visualização, (7), dos resultados da amostragem especificada. $\mathrm{Na}$ etapa (8), permite inserir mudanças no setpoint no intuito de avaliar o comportamento regulatório do controlador.

\subsection{Aplicação da Ferramenta PID em VBA}

Para avaliar o funcionamento e aplicabilidade da ferramenta de controle PID desenvolvida em VBA, foi utilizada a malha de controle para a pressão da coluna depentanizadora. A pressão de operação da mesma era de 9,02 bar, onde foi aplicado um degrau de $2 \%$ no valor nominal de operação. Os parâmetros de sintonia utilizados foram: $k_{c}=$ $1 \% / \%, \tau_{i}=20 \mathrm{~min}$ e $\tau_{d}=1 \mathrm{~min}$. Os resultados aplicando os controladores PID no VBA e no Aspen Dynamic $®$ estão apresentados na Figura 6. 

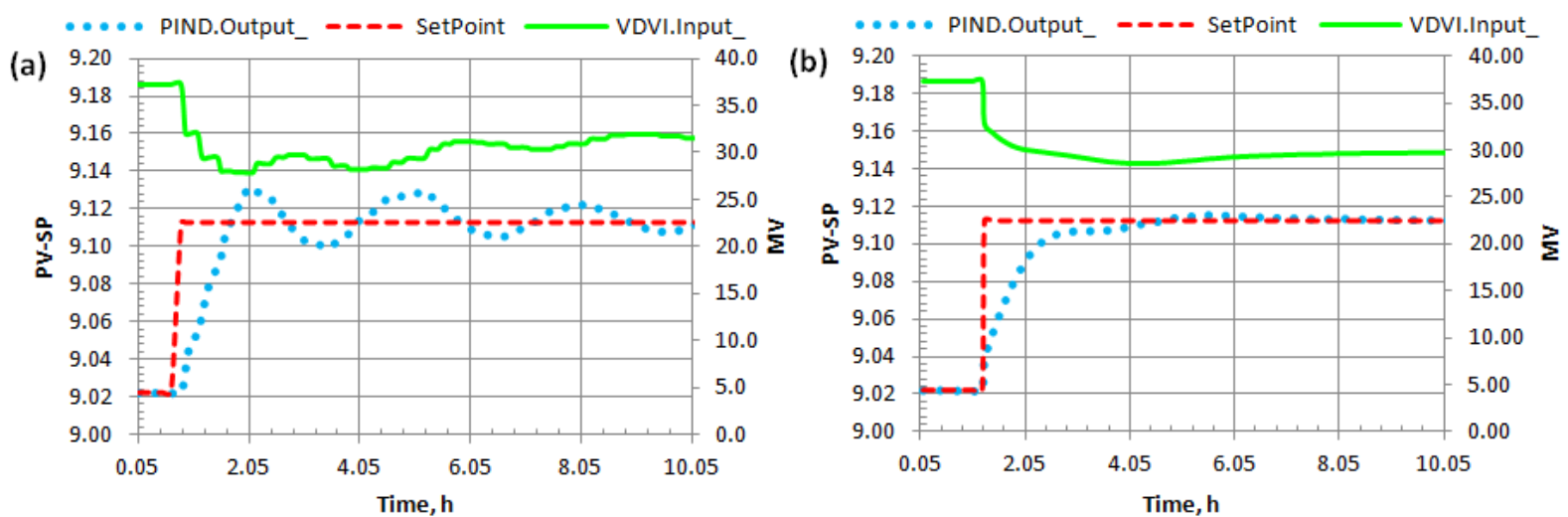

Figura 6 - Controle de pressão: (a) Ferramenta VBA; (b) Ferramenta Aspen®.

Para avaliação dos sistemas de controle em termos de desempenho, dentre os índices baseado nos erros, o utilizado foi o ITAE (Integrated of the Time multiplied by Absolute Error), sendo seus valores para o controlador desenvolvido e do Aspen foram: 1.999 e 0.7858, respectivamente. Pelos gráficos, tais valores são corroborados, onde o melhor desempenho foi obtido com o controlador do próprio Aspen. Tal desempenho é motivado pelo algoritmo utilizado no Aspen ser modificado do algoritmo clássico, o qual foi tomado como referência neste trabalho.

\section{CONCLUSÃO}

A Ferramenta desenvolvida apresentou resultados satisfatórios quanto à capacidade de controlar a variável de pressão da coluna utilizada no estudo. O mesmo pode ser obtido para as demais variáveis de processo, uma vez que devido às limitações de espaço não foram possíveis de apresentar nesse trabalho.

Apesar do desempenho de sido inferior, quando comparada com a ferramenta de controle padrão do Aspen Dynamic, a mesma se mostra promissora nos estudos iniciais de controle de processos, onde os detalhes do algoritmo clássico PID podem ser avaliados nos seus detalhes computacionais.

\section{REFERÊNCIAS BIBLIOGRÁFICAS}

ASTRÖM, K. J., Process Control. Lecture 3. Department of Automatic Control LTH,LundUniversity,Disponívelem:http://www.control.lth.se/media/Education/DoctoratePro gram/2012/HistoryOfControl/L03ProcessControleight.pdf, acessado em 10 de setembro de 2013.

SVRCEK, W. Y.; MAHONEY, D. P.; YOUNG, B. R., A Real-Time Approach to Process Control, 2nd edition, John Wiley \& Sons, 2006.

LUYBEN, W. L.; LUYBEN, M. L., Essentials of process control, New York: McGraw-Hill, 1997.

LUYBEN, W.L. Process Modeling, Simulation and Control for Chemical Engineers. 2nd ed, McGraw-Hill International Editions, 1990.

STEPHANOPOUlOS, G., Chemical Process Control: An Introduction to Theory and Practice, Englewood Cliffs, N.J.: Prentice Hall, 1984. 